\section{The metabolic syndrome predicts cardiovascular prognosis}

The metabolic syndrome is characterized by a grouping of metabolic risk factors in an individual, which can include obesity, high blood pressure, and glucose and lipid disturbances. A link between the metabolic syndrome and increased risk of cardiovascular disease (CVD) has been shown in several studies, and guidelines now include the syndrome as a risk factor for CVD. An article published in the BMJ adds to these data, listing the metabolic syndrome as a prognostic indicator of total and cardiovascular mortality.

Sundström and co-workers in Uppsala, Sweden, enrolled a large community-based group of 50-year-old men, to investigate whether the presence of the metabolic syndrome increased the risk of total and cardiovascular mortality when added to models of established risk factors for CVD (smoking, diabetes, hypertension and serum cholesterol). They used modified versions of the National Heart, Lung, and Blood Institute National Cholesterol Education Program (NCEP) and WHO definitions to define the metabolic syndrome.

At 50 years of age, presence or absence of the metabolic syndrome was predictive of total and cardiovascular mortality according to both definitions, although the NCEP definition was more strongly predictive; individuals with the metabolic syndrome had a 40-60\% increased risk of total and cardiovascular mortality. At 70 years of age, however, the syndrome was not a reliable prognostic indicator. The authors advise that additional studies of a similar nature be done to confirm their findings.

Original article Sundström J et al. (2006) Clinical value of the metabolic syndrome for long term prediction of total and cardiovascular mortality: prospective, population based cohort study. BMJ 332: 878-882

\section{Candesartan is effective in treating prehypertension}

Many people with prehypertension go on to develop hypertension. Prehypertension is associated with excess morbidity and deaths, as well as other cardiovascular risk factors. The Trial of Preventing Hypertension (TROPHY) attempted to determine whether the development of hypertension could be prevented or delayed by the treatment of prehypertension.

Participants classified as having prehypertension $(130-139 / \leq 89 \mathrm{mmHg}$ or $\leq 139 / 85$ $89 \mathrm{mmHg}$ ) were randomly assigned to receive either $16 \mathrm{mg}$ of the angiotensin-receptor blocker candesartan or placebo daily for 2 years. At the end of this period, hypertension had developed in 53 of 391 (13.6\%) patients in the candesartan group and in 154 of 381 (40.4\%) patients in the placebo group $(P<0.001)$, equating to a relative risk of $0.34(95 \% \mathrm{Cl} 0.25-0.44)$ for the candesartan group. After 2 further years in which both groups received placebo, incidence of hypertension remained lower in the candesartan group: hypertension developed in 208 (53.2\%) patients in this group compared with 240 (63.0\%) patients in the placebo group, indicating a relative risk of $0.84(95 \% \mathrm{Cl} 0.75-0.95)$ for the candesartan-treated patients.

Previous studies have shown that individuals with prehypertension are at increased risk of coronary disease and stroke; the findings in this study suggest that pharmacotherapy for the control of prehypertension is an effective and well-tolerated way to reduce this risk. The authors state that lifestyle modification measures for the control of blood pressure have had limited effect, and suggest a cost-effectiveness comparison of pharmacotherapy versus lifestyle modification for blood pressure control.

Original article Julius S et al. (2006) Feasibility of treating prehypertension with an angiotensin-receptor blocker. N Engl J Med 354: 1685-1697

\section{Imaging by 64-slice CT accurately diagnoses stenosis in CABG}

Invasive coronary angiography (ICA) is the standard method for follow-up examination after CABG but is limited by discomfort to patients, high cost and risk of complications. A noninvasive alternative has therefore been sought. Angiographic studies of 64-slice CT have shown accuracy in the diagnosis of coronary artery stenosis. Pache et al. therefore assessed the effectiveness of this modality in the diagnostic assessment of CABG, using ICA as a reference standard.

The researchers evaluated 96 bypass grafts in 31 patients. For the diagnosis of significant (>50\%) stenosis, 64 -slice CT had an overall 